\title{
Using Digital Imaging to Assess Spectral Solar-Optical Properties of Complex Fenestration Materials: A New Approach in Video- Goniophotometry
}

Marilyne Andersen*, Eleanor Stokes, Nicholas Gayeski, Courtney Browne

Building Technology Program, Department of Architecture, Massachusetts Institute of Technology, Cambridge MA, USA

\begin{abstract}
A large variety of angularly selective fenestration systems has been developed in the past two decades and shows great potential in improving visual comfort while reducing energy consumption, especially when combined with spectrally selective properties. Such systems include light-redirecting glazing, shading, film coatings, reflectors and others. To assess the potential of these systems accurately and reliably, one needs to be able to predict in detail how they modify the energy, direction, and spectral make-up of solar radiation. For this assessment, spectral (wavelength-dependent) Bidirectional Transmission or Reflection Distribution Functions are used, usually referred to as BTDFs or BRDFs, or more generally BSDFs for Scattering Functions.

To enable a faster, cheaper, and continuous investigation of these properties over most of the solar spectrum (400 to $1700 \mathrm{~nm}$ ), an innovative goniospectrometric instrument has been created, relying on digital imaging, on light collection by an ellipsoidal half-transparent mirror, and on a filtering method in the visible range to generate spectral radiometric BSDFs. This so-called Heliodome instrument is described in this paper. It enables the performance of new fenestration technologies to be assessed in terms of lighting and solar gains management potential. The rotating table also serves as a heliodon, an architectural design tool for visualizing sunlight distribution inside a scale model and performing analyses on appropriate sun control strategies. The Heliodome's major innovations compared to other devices are to enable an analysis of both the visible and the near-infrared portions of the solar spectrum, to provide
\end{abstract}

\footnotetext{
* Corresponding author: Prof. Marilyne Andersen, MIT Building 5-418, 77 Massachusetts Avenue, Cambridge, MA 02139, USA. Phone: +1 617253 7714. Fax: +1617 253 6152. Email: $\underline{\text { mand@mit.edu }}$
} 
spectral as well as photometric light distribution data, and to ensure a continuous investigation of the transmitted or reflected light in a time-efficient way.

Keywords: digital imaging, bidirectional distribution function, solar spectrum, energy, complex fenestration systems, goniophotometer

\section{Introduction}

Any daylighting strategy has to be approached from an integrated perspective, encompassing its potential for illumination, heating, and pleasantness, and considering its liabilities in terms of glare, thermal discomfort, extra cooling needs, and variability. Indeed, as soon as daylight enters a space, so does solar radiation, either as a diffuse energy source (sky radiation) or as a concentrated energy input (direct sunlight). This also means that most daylighting strategies will affect lighting as well as heating and cooling, which altogether account for half of the building's energy requirements and about $20 \%$ of total U.S. energy consumption (U.S Department of Energy, 2006).

There is also more to daylighting than its energy savings potential. It is unquestionably one of the fundamental components of architecture(Lam, 1986)(Guzowski, 2000) and is highly relevant to cutting edge societal issues such as sustainability, well-being, health and - potentially - productivity (Cuttle, 2002) (Boyce, et al., 2003) (Heschong-Mahone Group, Inc., 1999) (Heschong-Mahone Group, Inc., 2003) (Heschong-Mahone Group, Inc., 1999) (Veitch, 2005) (Webb, 2006) (Brainard, et al., 2001) (Lockley, et al., 2006) (Pechacek, et al., 2008).

But, the benefits of daylighting are also highly sensitive to careful planning and control, and can only be achieved if visual and thermal comfort are ensured. Not only must we try to increase the availability and use of daylight inside buildings, we must also ensure its quality and therefore its energy-efficient and user-responsive control. The latter requires a careful daylighting strategy based on accurate predictions, for which detailed transmission and reflection data of the fenestration components are essential.

As a consequence, the control of daylight and solar radiation through fenestration systems has received growing attention both in research and practice for the past two decades and has led to the development 
of a large variety of innovative fenestration systems. These include complex glazing and shading systems and devices for redirecting the direct (sun) and diffuse (sky) components of natural light(Sullivan, et al., 1998)(International Energy Agency (IEA), 2000) (Kischkoweit-Lopin, 2002) (Koester, 2004) (Arasteh, et al., 2003). Often they integrate an angular redirecting component, such as in reflective blinds or prismatic panels, with a spectrally selective component, such as in spectrally selective films. The objective pursued for most of these systems is, typically, to refine the control of solar radiation under different seasonal and climatic conditions and/or deepen daylight penetration.

Their effective implementation in buildings is still uncommon, albeit expanding in high-end architecture (especially in Europe) (Koester, 2004) (Carmody, et al., 2004); this might seem surprising given their great potential in improving visual comfort and reducing energy consumption (one study estimates energy loads be reduced by $18 \%$ and electricity loads by $15 \%$ relative to spectrally selective glazings (Sullivan, et al., 1998)), or glazings with a solar control, low-e coating.

There are two reasons for this. One is their higher upfront cost, which will eventually be lowered through increased production. The other reason - influencing the first - lies in the arduous prediction of their performance and benefits for a given space, making their optimization process and their judicious selection particularly difficult. This is worsened by the fact that these emerging technologies are still distributed between research and commercial stages (Carmody, et al., 2004) without a comprehensive and structured database available for reference; in addition, their optical properties cannot be easily or rapidly assessed due to the many directional and spectral variables involved: climatic, diurnal and latitudinal variations, space parameters and more.

Because these systems are usually both spectrally and angularly selective, it is important to describe how solar radiation will be transmitted and/or reflected by them, in terms of energy, direction and spectrum. For this, spectral (wavelength-dependent) Bidirectional Transmission or Reflection Distribution Functions are used, defined as ratios between the radiance $L_{e}$ (or luminance $L$ ) emerging from the sample after either transmission or reflection of incoming light, and the irradiance $E_{e}$ (or illuminance $E$ ) received by the sample (Commission Internationale de l'Eclairage (CIE), 1977). The functions are usually referred to as BTDFs or BRDFs, or more generally BSDFs for Bidirectional Scattering Distribution Functions. 
The experimental assessment of spectral radiometric BSDFs, which, in their partial derivative form can be referred to as $\mathrm{BSDF}_{\mathrm{e}, \lambda}$, thus requires the ability to measure the spectral distribution of radiance at each emerging direction as well as the spectral irradiance of the material for any given incident direction. Instruments capable of performing such measurements are called bidirectional goniospectroradiometers or, if only photometric quantities are assessed, goniophotometers (Andersen, et al., 2006).

The latter are more common than the former and produce photometric BTDF and/or BRDFs over the visible range i.e. functions that account for the human eye's sensitivity to wavelengths and integrate light spectra over the photopic response $\mathrm{V}(\lambda)$. Hence, they do not offer any spectral information and rely on a variety of approaches, mostly based on the scanning of all the emerging directions of light (Andersen, et al., 2006).

Only two devices, also based on scanning methods, allow fenestration systems to be characterized spectrally, described in (Breitenbach, et al., 2001) and (Apian-Bennewitz, 2007), with a possibility to extend the wavelength to NIR for the latter. Because scanning cannot avoid the risk of missing transmission or reflection features between measured points, this approach often requires refinements in resolution for high light gradients and can thus become a time-consuming process.

Projection-based approaches, relying on calibrated digital cameras, have been developed as alternatives (Andersen, et al., 2005)(Ward, 1992) but have so far not covered wavelength ranges beyond the visible nor enabled spectral analysis.

For fenestration systems that are simultaneously angular and spectral, BSDF $F_{e, \lambda}$ (e (subscript) denotes a radiometric quantity, $\lambda$ (subscript) denotes a spectral quantity) become necessary because these complex fenestration samples could modify the spectral power distribution of incident light differently for different emerging angles. In addition, discarding the spectral component means that BSDF results will only be valid in characterizing a sample for a particular incident spectrum; in other words, the relative spectra of the light source used for measurement and that of the light source used in actual application must be very similar in order to achieve meaningful results. 
A new kind of goniospectroradiometer, called Heliodome and based on digital imaging techniques, was created to enable a faster, cheaper and continuous investigation of quasi-spectral radiometric BSDFs over most of the solar spectrum (400 to $1700 \mathrm{~nm}$ ). This paper describes its functioning principle (and dual application as a sun course simulator), its major components and calibration procedures, and the sequence of processing stages necessary to achieve reliable $\mathrm{BSDF}_{\mathrm{e}, \lambda}$ values for the evaluation of the lighting and solar gains management potential of new fenestration technologies.

\section{Functioning principle of the Heliodome}

The functioning principle of the Heliodome is illustrated in Figure 1. As formally expressed by Equation 1, the overall aim of a BSDF measurement is to determine how much radiation is re-directed by a material sample in each direction, for a given incident angle.

$$
\operatorname{BSDF}_{e, \lambda}\left(\theta_{i}, \varphi_{i}, \theta_{r}, \varphi_{r}\right)=\frac{L_{e, \lambda}\left(\theta_{i}, \varphi_{i}, \theta_{r}, \varphi_{r}\right)}{E_{e, \lambda}\left(\theta_{i}\right)}
$$

where $\left(\theta_{\mathrm{i}}, \phi_{\mathrm{i}}\right)$ are the polar coordinates of the incident beam, and $\left(\theta_{\mathrm{r}}, \phi_{\mathrm{r}}\right)$ are the polar coordinates of emerging radiation, with $\theta$ referring to altitude angle $\left(^{\circ}\right)$ and $\phi$ to the azimuth angle $\left({ }^{\circ}\right) ; L_{e, \lambda}\left(\theta_{i}, \phi_{i}, \theta_{r}, \phi_{r}\right)$ is the spectral bidirectional radiance emerging from the sample $\left(\mu \mathrm{W} / \mathrm{m}^{2}-\mathrm{sr}\right)$ in each direction ; and $\mathrm{E}_{\mathrm{e}, \lambda}\left(\theta_{\mathrm{i}}, \phi_{\mathrm{i}}\right)$ is the spectral irradiance on the sample plane $\left(\mu \mathrm{W} / \mathrm{m}^{2}\right)$ due to the incident beam's flux .

Hence, instead of moving a light sensor from one position to the next so as to analyze light distribution over the entire transmission or reflection hemisphere (scanning approach, see (Andersen, et al., 2006) for further details), here light emerging from the sample is first collected by an optical surface and digital images of that surface are captured by a camera that has been previously calibrated to act as a multiplepoints spectro-radiometer (see section 5). To make it possible to view all the emerging directions at once, the light-collecting surface is a hemi-spheroid ${ }^{\dagger}$, the camera and the sample are placed at its two foci, and the camera is equipped with a fish-eye lens to visualize the entire hemi-spheroid from the inside (180 degree opening angle).

\footnotetext{
${ }^{\dagger}$ A spheroid is an ellipsoid in which two of the three axes are equal; it is generated by rotating an ellipse around one of its axes.
} 
FIGURE 1 HERE

This concept had been developed for a prototype goniophotometer designed to perform reflection measurements for computer graphics applications in the early nineties (Ward, 1992), and used a mirrored hemisphere as the light-collecting surface. For the Heliodome a hemi-spheroid was designed instead.

Achieving this more ideal geometry with enough optical accuracy turned out to be a huge issue in terms of feasibility, but was ultimately overcome (cf. Section 4).

Two digital cameras are actually used so as to cover most of the solar spectrum in terms of wavelength range: a Charge Coupled Device (CCD) camera to cover the visible range (380 $\mathrm{nm}$ to about $1100 \mathrm{~nm}$ ), and an Indium Gallium Arsenide (InGaAs) camera for the Near-Infrared (NIR) (900 nm to $1700 \mathrm{~nm}$. Their calibration and use as spectro-radiometers is described in section 5.

The hemi-spheroid, the sample and either camera are all secured on a motorized rotating table, described in further detail in section 3. The table has two degrees of rotational freedom and is controlled by two separate motors. The sample is illuminated by a fixed light source and is located at the center of the table, which also corresponds to one of the foci of the hemi-spheroid. By varying the tilt and azimuth angles of the table (and hence of the sample), any incident direction can be achieved.

The light source is a 400W "Dedolight" HMI projector chosen because of its collimation and its relative emittance spectrum, which is reasonably similar to that of sunlight (see Figure 5(a)). The beam diameter is adaptable through an aperture that varies the size of the output port of the lamp, which greatly affects the illuminance on the sample plane and the angular spread of the beam. The beam's half angular spread was chosen to be $2.4^{\circ}$ to minimize errors due to beam spread and variance.

For reflection measurements, the incident light beam must pass through the spheroid's semi-transparent shell before reaching the sample itself, causing the beam to no longer be restricted to the sample area. 
Light reflections from the illuminated area outside of the sample's diameter can contribute to errors in the camera's output. To ameliorate this problem, in reflection, the beam has been modified with a black light corset, fixing the beam diameter such that it only illuminates the sample (parasitic light error was not quantified because all that is left after the corset is applied are the two spots resulting from initial reflection and scattering of the incident beam on the dome..

In transmission, the incident beam comes from the opposite side of the sample, where there is no dome but where the camera is fixed (see Figure 1). Hence, the camera might obstruct incident light for a small range of azimuth angles. For the few cases where the sample's heterogeneity makes this critical, it is rotated $90^{\circ}$ on its holder to fully characterize it within the missing angular range.

Light emerging from the sample, at one focal point, after either transmission or reflection then gets reflected once off of the hemi-spheroid to the digital camera (at the other focal point).

For every incident direction, a set of images are taken at various exposure times and converted into radiance maps to produce a High-Dynamic Range (HDR) image of the interior of the spheroid. Sections 5 and 7 explain how the raw image information, i.e. its pixels' digital levels ( $R, G$ and $B$ channels for the CCD camera, and a unique channel for the InGaAs camera) are converted into spectral radiance values. It describes the set of spectro-radiometric calibration procedures that first have to be applied, and how incoming light is filtered into smaller wavelength intervals so as to extract spectral information from the images.

Pixel coordinates on the images are then related to directions along which light emerges from the sample; this relationship depends on the geometry of the spheroid and the optical properties of the fish-eye lens. Starting from the irradiance at the sample plane and accounting for the fact that light is first reflected on the spheroid's surface before being detected by the camera, spectral radiometric BSDFs can be deduced from the wavelength-dependent radiance matrices based on a refined spectral estimation method that assesses the HDR images, as described in section 5.3.

\section{Mechanical base of the Heliodome}


Two major structural elements make up the heliodome's platform, shown on Figure 2(e). The first is an octagonal frame made of 8 identical hollow aluminum tubes of square section, welded together, that rotates around the horizontal axis. The second is a $1.5 \mathrm{~m}$ diameter disk that rotates around its normal by the way of peripheral gears thanks to three pairs of rollers mounted on the top and bottom of the frame (Fig. 2(c)). They include roller ball bearings to center the disk in the plane of the octagonal frame. The spur gears responsible for the azimuth rotation of the disk are fixed on the octagonal frame (Fig. 2(b)).

The disk consists of a hexagonal frame assembly made out of Unistrut ${ }^{\circ}$ rails and of a set of 6 curved trapezoidal pieces of aluminum honeycomb (Fig. 2(a)).

The device accommodates samples whose dimensions are between $100 \mathrm{~mm}$ and $200 \mathrm{~mm}$ in diameter (or in diagonal dimension), with thicknesses less than $20 \mathrm{~mm}$. As illustrated in Figure 2(d), the sample holder consists of a pair of diaphragms that holds the sample in place by compression ("sandwich" system) thanks to three springs of adjustable height. These springs are inserted into bolts fixed to the central part

of the Unistrut ${ }^{\circledR}$ frame. As the diaphragms cannot be more than $1 \mathrm{~mm}$ thick because shadows would become significant at grazing angles, a second pair of thicker diaphragms is added on top and bottom of the thinner ones for reinforcement, presenting apertures large enough to avoid producing shadows on the sample.

The same supporting platform is also used as an educational tool: it can be converted into an automated, computer-controlled heliodon for sunlight penetration analyses on scale models (Osser, et al., 2007). This dual application imposed some additional constraints on the table construction, such as enabling clamps and a camera holder to slide along the rails and secure scale model bases of varying dimensions.

FIGURE 2 HERE

\section{A semi-transparent hemi-spheroid for reliable light collection}

The hemi-spheroid is an acrylic shell coated with a thin film of aluminum, such that it is about $40 \%$ transmissive over the whole dome. Its interior dimensions are $1150 \mathrm{~mm}$ (major axis) $\mathrm{x} 1122.5 \mathrm{~mm}$ (minor 
axis) such that the two focal points are about $200 \mathrm{~mm}$ apart. This combination of dimensions made it possible to have the camera holder next to the sample holder, but far enough away to prevent light from being directly emitted from the sample to the camera. The combination also was chosen to maintain a difference between the semi-major axis and the focal radius always longer than five times the sample diameter, at which distance the sample and camera can be approximated as a single point according to the optical properties of using a hemispheroid.

The dome is fixed on the rotating table using an asymmetrical flange at the base (see Figure 3(a)). The smaller width of the flange on one side ensures that the rollers on Fig. 2(c) never touch any part on the spheroid and a pair of holes in the flange, which define the minor axis, are used to precisely position the dome on the table.

As the hemi-spheroid is the vehicle for radiation traveling from the sample to the camera, it is most important that the surface and geometry be pristine. The geometrical accuracy, surface characteristics, and spectral properties of the spheroid greatly affect the resulting imaging, and thus are critical in the accurate estimation of spectral BSDFs.

After unsuccessful attempts to achieve enough accuracy with conventional thermoforming processes (cast and extruded domes with a custom-made male mold - the dome shown in Figure 1 was one of the resulting ones), the resort to a free-blowing method was tested.

The final hemi-spheriod was made of mirror grade extruded acrylic, which was free-formed in a vacuum cavity at Replex Plastics, Inc; it is shown in Figure 9(b). The acrylic was heated with differing heat patterns that altered the dome's shape which was controlled by the pressure differential across the bubble balanced by the tensile stress in the polymer.

The spheroid was then coated inside with a thin-film of aluminum through a cathodic arc evaporation physical vapor deposition (PVD) process.

As explained in section 5, a detailed knowledge of the hemi-spheroid's spectral reflectance and transmittance in the $380-1700 \mathrm{~nm}$ range is necessary to determine how it modifies the spectral power 
distribution of incident and emerging radiation; this information is indeed used to assess a sample's spectral transmittance or reflectance accurately, which is the ultimate goal of the Helidome. A thorough analysis of the dome's optical properties was therefore performed over its entire surface, assessing the spectral and total transmittance and reflectance every 5 degrees in altitude and every 45 degrees in azimuth; some of the results are shown in Figure 3(b) to (d). A USB2000 spectrometer was used for spectral measurements over the visible range, in combination with an integrating sphere and a calibrated silicon detector, and a NIR512 spectrometer for the near-infrared range, with the same integrating sphere but a calibrated InGaAs detector.

The results showed that the aluminum coating exhibited very constant transmittance properties for all azimuth angles and over both the visible and near-infrared ranges. Total transmittance decreased from $50 \%$ at the apex to about $35 \%$ (VIS) or $45 \%$ (NIR) at the flange, but the relative spectrum remained approximately the same.

The spectral reflectance of the spheroid was also measured for varying altitude and azimuth angles and because it was difficult to achieve enough accuracy with absolute spectral measurements, a functional relationship between transmittance and reflectance was determined, based on the almost linear relationship revealed in Figure 3(b).

As the spectral transmittance was measured to be uniform along any given altitude, the transmittance (and thus reflectance) of the spheroid to $500 \mathrm{~nm}$ radiation could be estimated over the entire surface of the spheroid for the given altitude angles by interpolating between the major and minor axis measurements. The resulting weights, shown in Figure 3(c) and (d), are used to scale the relative spectrum to find the spectral transmittance or reflectance for any incident angle on the entire spheroid. This $\rho_{\text {spheroid }, \lambda}\left(\theta_{\mathrm{i}}, \phi_{\mathrm{i}}\right)$ and $\tau_{\text {spheroid }, \lambda}\left(\theta_{\mathrm{i}}, \phi_{\mathrm{i}}\right)$ will be relied upon extensively in the spectral estimation method described in section 5.3 .

FIGURE 3 HERE

\section{Converting digital cameras into multiple-points spectro-radiometers}


Two digital cameras are used in the Helidome, a Kappa DX20 color CCD camera sensitive in the $380 \mathrm{~nm}$ to $950 \mathrm{~nm}$ spectral range and a Sensors Unlimited SU320 1.7RT near-infrared camera sensitive in the 900 to $1700 \mathrm{~nm}$ range. Both utilize an interchangeable Fujinon high resolution fisheye lens $\left(185^{\circ}\right.$ opening angle).

Once light has been collected by the spheroid and reflected back to the cameras, the major remaining challenge is to convert each of these cameras into multiple-points spectro-radiometers. A set of calibration procedures was thus applied for both digital cameras to provide the necessary link between the digital levels of the captured images (for adequately exposed pixels) and the corresponding scene radiances at a given emerging direction from the sample. These procedures are described in (Gayeski, et al., 2008) and are summarized below.

By combining the resulting radiances through HDR imaging (Inanici, 2006), the pixels of the HDR form a map of the emerging light distribution, with each pixel associated to a radiance and to the spectral reflectance or transmittance of the sample in a certain direction.

\subsection{Spectro-radiometric calibrations}

Upon capturing the reflectance of a monochromatic beam, the camera's response, evaluated as a normalized digital level $\left(N D L_{R, G, B}\right)$, was associated with a spectral exposure, $h(\lambda)$, through a logistic dose response function given by Equation 2:

$$
\mathrm{NDL}_{\mathrm{R}, \mathrm{G}, \mathrm{B}}=\alpha+\frac{\beta}{1+\left(\frac{\mathrm{h}(\lambda) / \mathrm{h}_{\mathrm{R}, \mathrm{G}, \mathrm{B}}^{0.3}(\lambda)}{\gamma}\right)^{\delta}}
$$

where $\alpha, \beta, \gamma, \delta$ are experimentally derived constants and $\mathrm{h}_{\mathrm{R}, \mathrm{G}, \mathrm{B}}^{0.3}(\lambda)$ is the spectral exposure required to yield a normalized digital level of 0.3 (Gayeski, et al., 2008). To measure the relative response of the camera across different wavelengths, it is only necessary to compare $h^{0.3}{ }_{R, G, B}(\lambda)$. 
This response of the camera to different wavelengths in different channels is called the absolute spectral responsivity (ASR), $\mathrm{r}_{\mathrm{RGB}}(\lambda)$, where

$$
r_{R, G, B}(\lambda)=1 / h_{R, G, B}^{0.3}(\lambda)
$$

The ASR for the CCD camera and the InGaAs camera have been experimentally determined by imaging the cameras' response to monochromatic beam spots of measured radiances (Gayeski, et al., 2008), and are shown for the Red, Green, and Blue channels and for the unique NIR channel in Figure 4.

\section{FIGURE 4 HERE}

For polychromatic radiation such as natural daylight, the ASR curves must be weighted by the relative spectrum of the beam reaching the camera to give a new $h_{R, G, B, b e a m}^{0.3}$ Thus, by knowing the relative spectrum of the beam, and the digital output of the camera in at least one channel, it is possible to predict the total radiance of the beam, $L_{e, b e a m, 380-945}$, across the wavelength range where the camera is sensitive through Equation 4:

$$
\mathrm{L}_{\mathrm{e}, \text { beam }, 380-945}(\lambda)=\frac{\mathrm{h}_{\mathrm{R}, \mathrm{G}, \mathrm{B}}^{0.3}(\lambda)}{\mathrm{t}_{\mathrm{int}}} \times \gamma\left(\frac{\beta}{\left(\mathrm{NDL}_{\mathrm{R}, \mathrm{G}, \mathrm{B}}-\alpha\right)}-1\right)^{1 / \delta}
$$

If the relative spectrum of the detected light is known, such as for a white wall illuminated by daylight after transmission through a clear pane of glass or when analyzing the optical properties of spectrally neutral samples, these spectroradiometric calibrations already allow the use of these digital cameras as extremely time-efficient, multiple points luminance- and radiometers. But in many instances, the relative spectrum of detected light will be unknown, which is typically the case for any spectrally-selective material whose transmission and reflection properties are yet to be determined.

Since the Heliodome is primarily designed to characterize the distribution of radiation through unknown fenestration samples, it is most important for the camera to measure radiances from unknown spectra, and to then provide some information about what can be inferred about a sample's reflection and 
transmission spectra. The filtering system that leads to the estimation of these quasi-spectral properties of an unknown sample, based on (Gayeski, et al., 2008), is summarized below. A refined spectral estimation method was then developed, described in section 5.3, that builds upon this information to derive a closer approximation to the reflectance or transmission spectra of the sample. For further details about this method, see (Stokes, et al., 2008).

\subsection{A filtering system for quasi-spectral estimations}

The objective of adding a filtering system for detected (or incoming) light is to split the unknown spectrum into wavelength intervals across which the above-described calibration procedures enable an accurate estimation of the total radiance, or in other words, over which the camera sensitivity is flat enough.

These wavebands have to be small enough to minimize the change in ASR due to a varying relative spectrum. They were defined such that even with the most variant spectra, i.e. monochromatic beams, the predicted radiance would still differ from the true radiance by no more than a defined amount.

To define these wavebands, a filterset consisting of Schott colored glass short and long pass filters, whose combined transmission curves are given in Figure 5(b), was chosen to best isolate the flatter sections of at least one channel's ASR for each waveband. This particular set of eight filterbands was found to be the best compromise between limiting the number of filters (and hence number of actions to perform during BSDF measurements) and ensuring reasonable accuracy. The NIR range was chosen to remain unfiltered because of its relatively flat sensitivity (see Figure 4(b)).

By designing the filters so that they transmit in each of these band ranges while filtering all other wavelength ranges, the approximate radiance in each band was isolated, and an approximation of the spectral power distribution of the light emerging from the sample was reconstructed.

FIGURE 5 HERE 
Before their combination into eight HDR images, the same pixel in each of the raw filterband images is analyzed at several exposure times to give a radiance step curve. Each step describes the radiance of the beam in a particular waveband $\Delta \lambda_{j}$ (with $j=1$ to 8 corresponding to each of the eight filtersets), which has been reflected from the corresponding location on the hemi-spheroid.

The response of the camera to detected light depends on the sample's optical properties but also on the incident beam (spectrum shown in Fig. 4(a)), the filters' and the spheroid optical properties (see section 4). Since these can all be determined through appropriate measurements and remain unchanged, the only unknown remains the sample's bidirectional spectral reflection or transmission coefficients, to be determined along each emerging direction (or for each pixel on the image) and for each incident direction.

A range of fenestration materials including coatings, applied films, monolithic substrates, and laminate combinations on clear and tinted glazings, were selected from the Optics 5 database (Lawrence Berkeley National Laboratory, 2008) to estimate errors in the camera estimated radiances for different samples. These errors were calculated within each filter set for radiance estimations, and for unfiltered radiation across the entire 380 to $945 \mathrm{~nm}$ wavelength interval for both luminance and total radiance estimations (Gayeski, et al., 2008). They were found to remain below $5 \%$ within each waveband for most spectra, and below $3 \%$ when estimating total radiance or luminance. Even when accounting for the $5 \%$ uncertainty that has to be introduced to account for camera calibration errors, this method therefore offers very satisfactory estimations of quasi-spectral radiance.

The resulting set of radiance values is actually a step function that consists of a series of average radiances describing the resultant beam after it has been transmitted through or reflected by the sample and reflected back to the fisheye lens. As the spectral properties of the dome are known, we can use this information to go back to the spectrum actually emitted by the sample, This means changing the radiance step curve derived from our measurements into a quasi-spectral step curve $\tau_{\text {box }}$ or $\rho_{\text {box }}$ that provides a rough estimation of the spectral transmittance or reflectance of the sample across the full wavelength range. Two examples of such a function are given in Figure 6. 
While this method provides accurate average transmission or reflection coefficients over specified wavebands, it can only recreate the gross spectral properties of fenestration samples (see Fig. 6).

This revealed the necessity to apply a refined spectral estimation method so as to more accurately predict the spectral reflectance or transmittance of unknown materials.

FIGURE 6 HERE

\subsection{Refinement into spectral transmittance and reflectance estimations}

One of the first aims of the spectral estimation method is to connect the above described step function to the sample's reflection and transmission spectra.

The method relies on the digital output of the CCD camera, i.e. the R, G, B values, for each of the eight filterbands described above to reconstruct a sample's spectrum. It approximates the latter by the truncated generalized singular value decomposition, and the errors are analyzed so as to choose the correct point of truncation (Stokes, et al., 2008).

The method was validated both experimentally and through simulations. The average improvements allowed by this refinement method over the "box-like" quasi-spectral assessment method were of $70 \%$ in the estimation of spectral reflectance and transmittance for the highly spectrally selective reference samples that were tested, and $40 \%$ for samples with flatter and smoother reflectance or transmittance spectra (for which the "box-like" approximation was already good). In the experimental validation, these improvements averaged $50 \%$. Figure 7 shows two spectrum examples that illustrate these results (Stokes, et al., 2008).

FIGURE 7 HERE

\section{Using the camera as a luminance-meter for unknown spectra}


While it would be easy to achieve photometric BSDFs from radiometric BSDFs, the photometric analysis would then become unnecessarily lengthy, capturing images for eight filterbands and all integration times while, in the end, results will be integrated over the visible spectrum anyway. It would therefore be much more time-efficient to create a calibration procedure that allows us to use the CCD camera differently depending on whether we want it to act as a spectro-radiometer or as a multiple-points luminance-meter; and the measurement time for photometric results would then be eight times faster than radiometric analyses.

A luminance calibration of the camera was thus performed to modify the camera's absolute spectral responsivity so that it more closely emulates the photopic function $V(\lambda)$ I.e the spectral sensitivity of the human eye.

\subsection{Adjusting the camera's spectral sensitivity}

The CCD camera's spectral sensitivity has been determined for all three channels R, G and B (see Figure 4) (Gayeski, et al., 2008). The first step in this photometric calibration is to deduce a unique number from the camera's R, G and B responses, such as done in (Inanici, 2006). An equal parts addition of the three response curves (see Figure $8(a)$ ) shows that the camera is overly sensitive in the red and blue portions of the spectrum compared to the targeted $V(\lambda)$ photopic function, and that there are two sensitivity peaks one around $530 \mathrm{~nm}$ from the green curve and one at 590nm from the red curve, both cradling the targeted $\mathrm{V}(\lambda)$ peak at $555 \mathrm{~nm}$.

To address these two issues, an optimization was performed, allowing autonomous scaled adjustments to the Red, Green, and Blue sensitivity curves so as to make their normalized, weighted sum as close to $\mathrm{V}(\lambda)$ as possible. To restrict the impinging signal to the photopic region, an assortment of Schott colored glass filters were also tested in pairs, optimizing their respective thicknesses to diminish the excessive sensitivity in the red and blue regions of the spectrum.

Two filters were chosen as a result of this optimization - GG495 at 2mm thickness, and BG40 at 2mm thickness. GG495, the long-pass filter, reduces the sensitivity in the low wavelength range, while BG40, a band-pass filter, gradually cuts out the signal from 560 to $700 \mathrm{~nm}$. 
In conjunction with new scaling parameters for the Red, Green, and Blue channels - which were 0.792, 0.657 , and 0 respectively (it was found that no weight should be assigned to the Blue channel in the weighted sum because the Green channel already has some excess blue sensitivity compared to $V(\lambda)$ ) (any contribution from the Blue channel would create premature spectral peaks whereas the Green channel includes this spectral band without overemphasizing it). The resulting corrected sensitivity $S(\lambda)$ is shown in Figure 8(b) together with the weighted $R, G$ and $B$ curves, $V(\lambda)$ and the transmission curves of the two filters. Albeit still imperfect, it is a great improvement over the initial uncorrected response.

The function $\mathrm{f}_{1}{ }^{\prime}$ (Commission Internationale de l'Eclairage (CIE), 1987) was used to quantify synchronization between $V(\lambda)$ and the new relative spectral sensitivity $S(\lambda)$, as shown in Equation 5 :

$$
f_{1}{ }^{\prime}=0.0093584 \cdot \sum|S(\lambda)-V(\lambda)| \cdot \Delta \lambda_{n}
$$

For the chosen filter combination, $f_{1}{ }^{\prime}$ went down to $17.6 \%$, whereas it was $99.7 \%$ for the uncorrected camera response.

\section{FIGURE 8 HERE}

Due to the disconcerting dip in $S(\lambda)$ around $570 \mathrm{~nm}$, a test was performed with hypothetical spectra to gauge the maximum error expected from this misalignment. The result of these tests suggested an upper limit for error of around $30 \%$ for the theoretical worst case of a box filter that would peak exactly at $570 \mathrm{~nm}$ (i.e. at the specific point of weakness for $S(\lambda)$ ). However, for the application of fenestration, expected error in luminance estimation remained lower than $10 \%$, which is comparable to other goniophotometric instruments (Andersen, et al., 2006).

\subsection{Photometric calibration to associate luminances with grayscale pixel values}

The purpose of the photometric calibration is to correlate grey scale pixel values (now from 0 to 369.5 due to the adjusted scaling parameters) with associated luminances for each integration time of the CCD camera. An experiment was set-up with the HMI light source illuminating a lambertian white diffuser 
(Spectralon ${ }^{\circledR}$ reflectance standard) through the designed cut-off filter pair described in the previous section. For each illumination setting, yielding luminances in the range of $1.55 \mathrm{Cd} / \mathrm{m}^{2}$ to $19,000 \mathrm{Cd} / \mathrm{m}^{2}$, images were taken at all integration times from detection to pixel saturation. The Red and Green channel's digital levels were then combined using the scalar weights described in the previous section to produce greyscale values that could be normalized and associated with the measured luminance levels.

The luminance calibration was validated with 5 spectrally selective fenestration samples used to alter the HMl's spectral power distribution in different ways; a Minolta luminance-meter was used for reference data. The difference between actual luminance and estimated luminance were calculated for over 100 images and all estimates fell within a 10\% error range of actual luminance measurements; the average error was $4.9 \%$. These results confirmed that the proposed photometric calibration was reliable enough to be useful in non-radiometric BSDF measurements.

\section{Estimating BSDFs for spectrally and angularly selective fenestration}

For every considered incidence direction $\left(\theta_{\mathrm{i}}, \phi_{\mathrm{i}}\right)$, the Heliodome's support platform is first tilted and rotated to illuminate the sample from the chosen incident angle. Starting at the source, the initial HMI beam is projected through one of nine Schott filter combinations - eight of which are used for radiometric BSDF assessment (section 5) and one which is used for photometric BSDF assessment (section 6), or through a hole if the near-infrared range is of interest. All of the filters are held in a filter wheel (see Figure 9(a)), which is programmed to cycle through the whole filterset once per incident angle while the camera captures a series of images at different integration times for each filter. The filtered beam then either directly hits the sample (transmission mode) or is first transmitted through the semi-transparent hemispheroid (reflection mode), shown in Figure 9(b). When leaving the sample, the transmitted or reflected light flux will be reflected off of the hemi-spheroid and redirected towards the camera port. Because the sample is not a point but a surface (and so is the camera's objective), the sample area needs to be constrained: a sample diameter of $10 \mathrm{~cm}$ was determined to be the minimal size that would still accurately redirect all incident beams such that their reflected spot completely covered camera port (Figure 9(d)). 


\section{FIGURE 9 HERE}

When the radiation reaches the camera, the resulting image is spatially calibrated so as to relate each pixel to a small area on the hemi-spheroid and each of these areas to its corresponding emerging direction $\left(\theta_{r}, \phi_{r}\right)$. Images are taken at different exposure levels (an example is shown in Figure 9(c)) to ultimately create a radiometric (or photometric) High Dynamic Range (HDR) image for each of the filter combinations. In Figure 9(e), the unfiltered light transmission through a holographic film (HOE) is shown and can be seen as the superposition of the 8 filter combinations given in Figure 9(f).

In a radiometric analysis, every adequately exposed pixel in each of these raw images has to be

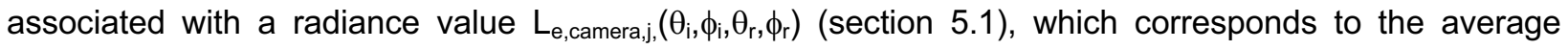
radiance impinging on the camera's lens for the current filterband (section 5.2). Filterbands 1 to 8 (whose boundaries are clearly visible in Figure $5(\mathrm{~b})$ ) and the full NIR range are used in radiometric analyses, whereas the photometric filterband will actually cover the full visible range.

For light-redirecting (angularly selective) systems, a BSDF is the formal quantity by which you can define their reflection or transmission coefficients (Commission Internationale de l'Eclairage (CIE), 1977). Hence, in either analysis mode, the quasi-spectral radiance value $L_{e, c a m e r a, j,}\left(\theta_{i}, \phi_{i}, \theta_{r}, \phi_{r}\right)$ can be used in the spectral estimation method described in section 5.3 to determine the spectral radiometric BSDF of the sample for that particular incident angle $\theta_{\mathrm{i}}, \phi_{\mathrm{l}}$ and for every pixel on the image (i.e. for every emerging direction $\theta_{\mathrm{r}}, \phi_{\mathrm{r}}$ ). In other words, the resulting series of HDR images ultimately maps the entire emerging distribution of light (one BTDF or BRDF) for each considered incident beam angle.

\subsection{Full process}

To frame the whole process leading from image acquisition to deliverables, the flow-chart shown in Figure 10 maps the spectral radiometric (dark grey) and photometric BSDF (white) acquisition and processing methods. Any automation steps used in both processes are in light grey. 
Each step assumes an uncropped image size of $1380 \times 1028$ pixels. Images are taken in a range of integration times between $3.95 \mathrm{e}-5$ seconds and 3.7 seconds (a total of 35 integration times). Two computer control softwares are used to capture the digital images. The CCD camera uses Kappa ImageBase Control, through which integration time can be varied. All other controls for the CCD camera remain unchanged, as all settings on the camera must match their respective values at the time of the radiometric calibration. The NIR camera is currently controlled with a National Instruments (IMAQ) card. Each phase necessary to achieve BSDF results was programmed in MATLAB® to achieve a full automation of the photometric and spectral radiometric BSDF assessment. These also include correction procedures, such as elimination of deviant pixels and of parasitic light, vignetting correction (Gayeski, 2007), and concentration and magnification effects of the spheroidal mirror that were assessed through ray-tracing simulations. Other sources of error, noted at the bottom right corner of the flow-chart, were investigated either experimentally, with ray-tracing simulations or both: they related to the noise to signal ratio, the polarization of light after its reflection off of the hemi-spheroid mirror, the point spread function, the variations within the HMI beam and were typically found to be either insignificant or not appropriate for systematic correction, in which case they were embedded in the overall error estimation for BSDFs.

When all of the images' raw data for each filter set have been corrected, they can be combined into 8 HDR images via the spectro-radiometric calibration described in section 5.1 (Gayeski, et al., 2008). Each HDR image is associated with three estimations of radiance, one in each of the Red, Green, and Blue channels. Thus the output at this stage is 24 radiance estimations for each pixel.

For each of the $1380 \times 1028$ pixels, the spectrum estimation method is then applied, which results in a reflectance (BRDF) or transmittance (BTDF) spectrum for the sample, $\rho_{k, \lambda}$, at each pixel. All the pixels are correlated to specific incident and emerging direction pairs via a spatial calibration (Gayeski, 2007) and can be grouped over chosen solid units of angle, and their associated BSDF values averaged, for each wavelength in $5 \mathrm{~nm}$ increments.

FIGURE 10 HERE

\subsection{Output}


Ultimately, two deliverables are produced for each incident angle. First, an $\mathrm{m} \times \mathrm{n} \times 114$ element matrix describing the spectral transmittance (BTDF) or reflectance (BRDF) of the sample at each of $m \times n$ emerging direction pairs $\left(\theta_{r}, \phi_{r}\right)$ for the considered incidence $\left(\theta_{i}, \phi_{i}\right) ; 114$ is the number of increments the visible range has been divided into ( $5 \mathrm{~nm}$ increments). If a NIR analysis was also performed, the matrix will extend to $m \times n \times 115$. If only a photometric analysis was performed, the matrix reduces to $m \times n \times 1$. In addition, a 3D spectral radiometric solid is created for a more intuitive and efficient visualization of the results than in matrix form.

These solids graphically combine angular and spectral information about the reflectance or transmittance properties of the sample by resorting to 3D plots similar to (Andersen, 2002) but using the color scale to represent spectrum rather than BSDF value, as this information is already contained in the spherical coordinates of the plotted data.

For each pixel group, the spectral reflectance or transmittance is converted into an RGB triplet. The RGB triplets are derived using a formula based on CIE chromaticities of the reference primaries (sRGB) (Commission Internationale de l'Eclairage, 1971). The sample is assumed to be illuminated by D65 illumination, a CIE daylighting standard (Billmeyer, et al., 1981). The final spectral radiometric solid thus shows the color of reflected or transmitted light for each angle, as well as the angular distribution of radiance. This provides a quite efficient way to describe how a sample spectrally modifies and distributes incident light throughout a space, and to reveal its Color Rendering Index (CRI) on a direction-dependent basis.

An example is shown in Figure 10(b) for the photometric solid and (c) for the radiometric solid respectively for a diffusing white paper and for an angularly and spectrally selective holographic film measured with this device. One can see that the photometric solid will show lighter colors as the BSDF increases in value whereas the colors in the radiometric plot will be determined by the spectral distribution. In both cases, the BSDF values will determine the spherical coordinates but these might be different depending on the graph type: for instance, while the radiometric BSDFs might be high for blue-related values and 
low for greenish ones, the equivalent photometric plot could show the opposite because it accounts for the photopic response $V(\lambda)$.

\section{Conclusions}

This paper describes a novel type of bidirectional goniospectroradiometer that uses a semi-transparent mirrored hemi-spheroid to redirect the emerging light from a material sample to a calibrated digital camera, either CCD or InGaAs, equipped with a fish-eye lens. This functioning principle minimizes the time required for a complete BSDF assessment, and enables a continuous assessment of the full distribution of light in reflection and transmission, over most of the solar spectrum (400nm to $1650 \mathrm{~nm}$ ) ,offering both photometric and spectroradiometric analysis options. This combination of capabilities is unprecedented in the field, which places this research in a leading position and makes the Heliodome an important instrument for the design and analysis of angularly and spectrally selective fenestration systems and their effective use of daylighting in a space.

This instrument is ultimately expected to be complemented by ray-tracing simulations for the characterization of whole daylighting systems or luminaires, restricting the measurements to their components.

One can see how this kind of detailed characterization can foster imaginative fenestration design. For example, incident light from different seasonal angles could be reorganized according to its spectral distribution - the NIR component in one direction (e.g. to a thermal mass) and the visible component in another direction (onto a reflective surface). Certainly, a better understanding of the complete BSDF and the directional reflectance or transmittance of complex fenestration systems will offer the possibility for greater control of solar radiation.

These functions, once measured, could help foster the increased use of complex fenestration systems. While BSDFs are used in a broad range of fields, from computer rendering to texture studies to luminaire characterization, in the field of daylighting, they are required for systems with angularly selective 
components. Knowledge of spectral BSDFs will aid fenestration designers to invent more efficient and holistically beneficial products, and will encourage the use of these products in the commercial sector by enabling architects and engineers to use the information to model CFS in their designs.

\section{Acknowledgements}

This work was jointly supported by MIT and the National Science Foundation under Grant No. 0533269. The authors would like to thank undergraduate students Dean Ljubicic, Timothy Koch, Danh Vo, Jason Ku, Samuel Kronick, Keith Molina, Javier Burgos and Zachary Clifford, Profs. Martin Culpepper and Leslie Norford as well as Steve Banzaert, Roselin Osser and Siân Kleindienst for their contribution and support to the project. They would also like to acknowledge Mark Schuetz from Replex Plastics, Michael Akkaoui from Tanury Industries, and Tim Dawsey from Spartech Corporation for their time and efforts dedicated to the making of the hemi-spheroid. Finally, they would like to acknowledge Lambda Research Corporation for having provided them with an educational license of TracePro.

\section{Nomenclature}

\begin{tabular}{|c|c|}
\hline $\mathrm{BSDF}_{\mathrm{e}, \lambda}$ & Spectral, radiometric Bidirectional Scattering Distribution Function $\left(\mathrm{sr}^{-1}\right)$ \\
\hline $\mathrm{E}_{\mathrm{e}, \lambda}\left(\theta_{\mathrm{i}}, \phi_{\mathrm{i}}\right)$ & Spectral irradiance on the sample plane due to the incident beam's flux $\left(\mu \mathrm{W} / \mathrm{m}^{2}\right)$ \\
\hline $\mathrm{f}_{1}^{\prime}$ & CIE (1987) measure of agreement between two spectra (percentage) \\
\hline$h(\lambda)$ & $\begin{array}{l}\text { Measured spectral exposure given by scene radiance multiplied by camera } \\
\text { integration time }\end{array}$ \\
\hline$h_{\mathrm{R}, \mathrm{G}, \mathrm{B}}^{0.3}(\lambda)$ & $\begin{array}{l}\text { Spectral exposure required to yield a normalized digital level of } 0.3 \text { in the CCD } \\
\text { camera }\end{array}$ \\
\hline $\mathrm{L}_{\mathrm{e}, \lambda}\left(\theta_{\mathrm{i}}, \phi_{\mathrm{i}}, \theta_{\mathrm{r}}, \phi_{\mathrm{r}}\right)$ & $\begin{array}{l}\text { Spectral bidirectional radiance emerging from the sample in each direction } \\
\left(\mu \mathrm{W} / \mathrm{m}^{2}-\mathrm{sr}\right)\end{array}$ \\
\hline $\mathrm{L}_{\mathrm{e}, \text { beam }, 380-945}(\lambda)$ & $\begin{array}{l}\text { Radiance of a polychromatic beam across wavelength range } 380-945 \mathrm{~nm} \\
\left(\mu \mathrm{W} / \mathrm{m}^{2}-\mathrm{sr}\right)\end{array}$ \\
\hline $\mathrm{L}_{\mathrm{e}, \mathrm{camer}, \mathrm{j}}\left(\theta_{\mathrm{i}}, \phi_{\mathrm{i}}, \theta_{\mathrm{r}}, \phi_{\mathrm{r}}\right)$ & Discretized spectral bidirectional filtered radiance seen by the camera $(\mu \mathrm{W} / \mathrm{n}$ \\
\hline
\end{tabular}




$\begin{array}{ll}N D L_{R, G, B} & \text { Normalized digital level (digital level divided by } 2^{8} \text { ) in the red, green, or blue } \\ & \text { channel } \\ r_{R, G, B}(\lambda) & \text { Absolute spectral responsivity of CCD camera in the red, green, and blue } \\ & \left.\left.\text { channel (NDL/( } \mu \mathrm{J} / \mathrm{m}^{2}-\mathrm{sr}\right)\right) \\ \mathrm{S}(\lambda) & \text { Modified camera sensitivity for photometric BSDF measurement } \\ t_{\text {int }} & \text { integration time for the camera (ms) } \\ V(\lambda) & \text { Photopic response function of human eye } \\ \alpha, \beta, \gamma, \delta & \text { Experimentally derived CCD camera calibration constants } \\ \Delta \lambda_{n} & \text { Arbitrary wavelength interval to assess the camera's corrected spectral sensitivity } \\ \Delta \lambda_{j} & \text { Wavelength interval corresponding to filterband } \mathrm{j} \text { for quasi-spectral estimation }\end{array}$

\section{References}

Andersen, M. (2002). Light distribution through advanced fenestration systems. Building Research and Information , 30 (4), 264-281.

Andersen, M., \& de Boer, J. (2006). Goniophotometry and assessment of bidirectional photometric properties of complex fenestration systems. Energy and Buildings , 38 (7), 836-848.

Andersen, M., Gayeski, N., Stokes, E., Osser, R., \& Browne, C. (2007). The Heliodome project: an innovative approach in assessing solar-optical properties of light-redirecting materials in combination with sun course simulations. Proceedings CISBAT 2007 - Renewables in a changing climate: Innovation in the Built Environment. Lausanne, September 4-5.

Andersen, M., Ljubicic, D., Browne, C., Kleindienst, S., \& Culpepper, M. (2005). Combining time-efficient goniophotometry with scale model studies in a unique instrument, In 2005, pp. , Berlin, September 19-21, . Proceedings of Lux Europa, (pp. 271-274). 
Andersen, M., Roecker, C., \& Scartezzini, J.-L. (2005). Design of a time-efficient video-goniophotometer combining bidirectional functions assessment in transmission and reflection. Solar Energy Materials and Solar Cells , 88 (1), 97-118.

Apian-Bennewitz, P. (2007). pab Goniophotometer II. Retrieved 07 05, 2007, from http://www.pabopto.de/gonio-photometer/

Arasteh, D., Apte, J., \& Huang, Y. (2003). Future Advanced Windows for Zero-Energy Homes. ASHRAE Transactions, 109, Part 2.

Billmeyer, F., \& Saltzman, M. (1981). Principles of Color Technology (2nd Edition ed.). New York: John Wiley \& Sons, Inc.

Boyce, P., Hunter, C., \& Howlett, O. (2003). The Benefits of Daylight. Rensellear Polytechnic Institute, Lighting Research Center. Troy, NY: Daylight Dividend Program.

Brainard, G., Hanifin, J., Greeson, J., Byrne, B., Glickman, G., Gerner, E., et al. (2001). Action Spectrum for Melatonin Regulation in Humans: Evidence for a Novel Circadian Photoreceptor. The Journal of Neuroscience , 21 (16), 6405-6412.

Breitenbach, J., Lart, S., Langle, I., \& Rosenfeld, J. (2001). Optical and thermal performance of glazing with integral venetian blinds. Energy and Buildings , 33 (5), 433-442.

Carmody, J., Selkowitz, S., Lee, E., Arasteh, D., \& Willmert, T. (2004). Window systems for highperformance buildings. Norton \& Company.

Commission Internationale de l'Eclairage (CIE). (1987). Methods of characterizing illuminance meters and luminance meters - Performance, characteristics and specifications. CIE 69.

Commission Internationale de l'Eclairage (CIE). (1977). Radiometric and photometric characteristics of materials and their measurement. CIE , 38 (TC-2.3).

Commission Internationale de l'Eclairage. (1971). Colorimetry: Official Recommendations of the International Commission on Illumination. CIE 15 (E-1.3.1). 
Cuttle, C. (2002). Identifying the human values associated with windows. International Daylighting, 5, 3-6.

Gayeski, N. (2007). New Methods for Measuring Spectral, Bi-directional Transmission and Reflection using Digital Cameras. M.Sc. Thesis, Massachusetts Institute of Technology, Building Technology Program, Department of Architecture, Cambridge, MA.

Gayeski, N., Stokes, E., \& Andersen, M. (2008). Using Digital Cameras as Quasi-Spectral Radiometers to Study Complex Fenestration Systems. Lighting Research and Technology, (submitted in December 2007).

Guzowski, M. (2000). Daylighting for Sustainable Design. McGraw-Hill.

Heschong-Mahone Group, Inc. (1999). Daylighting in Schools: An Investigation into the Relationship Between Daylighting and Human Performance. Fair Oaks, CA: California Board for Energy Efficiency Third Party Program.

Heschong-Mahone Group, Inc. (1999). Skylighting and Retail Sales: An Investigation into the Relationship between Daylighting and Human Performance. Fair Oaks, CA: California Board for Energy Efficiency Third Party Program.

Heschong-Mahone Group, Inc. (2003). Windows and Classrooms: A Study of Student Performance and the Indoor Environment. California Energy Commission.

Inanici, M. (2006). Evaluation of high dynamic range photography as a luminance data acquisition system. Lighting Research and Technology , 38 (2), 123-136.

International Energy Agency (IEA). (2000). Daylight in Buildings: A Source Book on Daylighting Systems and Components. IEA SHC 21 / ECBCS 29.

Kischkoweit-Lopin, M. (2002). An overview of daylighting systems. Solar Energy, 73 (2), 77-82.

Koester, H. (2004). Dynamic Daylighting Architecture: Basics, Systems, Projects. Birkhauser.

Lam, W. (1986). Sunlighting as Formgiver for Architecture. Van Nostrand Reinhold. 
Lockley, S., Evans, E., Scheer, F., Brainard, G., Czeisler, C., \& Aeschbach, D. (2006). Short-Wavelength Sensitivity for Direct Effects of Light on Alertness, Vigilance, and Waking Electroencephalogram in Humans. Sleep Physiology , 161-168.

Osser, R., Andersen, M., \& Norford, L. (2007). Development of Two Heliodon Systems and Recommendations for their Use. Proceedings of SOLAR 2007. Cleveland, July 7-12.

Pechacek, C., Andersen, M., \& Lockley, S. (2008). Combining Annual Daylight Simulation With Photobiology Data to Assess the Relative Circadian Efficacy of Interior Spaces. Proceedings of the eSim 2008 conference. Quebec City, May 20-22.

Lawrence Berkeley National Laboratory (2008). International Glazing Database.Downloadable from http://windows.lbl.gov/materials/IGDB/

Stokes, E., Gayeski, N., \& Andersen, M. (2008). Estimating Spectral Information of Complex Fenestration Systems in a Video-Goniophotometer. Lighting Research and Technology, In Press.

Sullivan, R., Beltran, L., Lee, E., Rubin, M., \& Selkowitz, S. (1998). Energy and Daylight Performance of Angular Selective Glazings. Lawrence Berkeley National Laboratory. U.S. Department of Energy.

U.S Department of Energy. (2006). 2006 Building Energy Databook. Retrieved 07 05, 2007, from http://buildingsdatabook.eren.doe.gov

Veitch, J. (2005). Light, Lighting, and Health: Issues for Consideration. LEUKOS , 2 (2), 85-96.

Ward, G. (1992). Measuring and Modeling Anisotropic Reflection. Computer Graphics , 26 (2), 265-272. Webb, A. (2006). Considerations for Lighting in the Built Environment: Non-Visual Effects of Light. Energy and Buildings , 38, 721-727. 


\section{FIGURE CAPTIONS}

Figure 1: Functioning principle of the Heliodome: The incident light beam reaches the material sample (at one focal point), from which reflected or transmitted light gets reflected once off of the hemi-spheroid to the digital camera (at the other focal point). Note: enhancement illumination was added under the dome for picture quality.

Figure 2. The Heliodome's mechanical support. (a) Assembly of the Table (b) Close-up of Spur Gears (c) Solid Model of Ball Bearing Rollers (d) Construction of Sandwich Locking Mechanism (e) Heliodome supporting table.

Figure 3. The hemi-spheroid's geometric and optical properties. (a) Major and minor axes (b) Relationship between reflectance and transmission for $500 \mathrm{~nm}$ light (c) Transmittance of spheroid to $500 \mathrm{~nm}$ radiation at all altitude and azimuth angles (d) Reflectance of spheroid to $500 \mathrm{~nm}$ radiation at all altitude and azimuth angles.

Figure 4: Absolute Spectral Responsivity of the two digital cameras. (a) CCD camera for the visible range, Red Green and Blue channels. (b) InGaAs camera for the near-infrared range, unique channel.

Figure 5. (a) HMI spectrum (b) Filterset spectra over the visible range

Figure 6. Real and predicted spectral transmittance $\tau_{\text {sample }}$ and quasi-spectral transmittance $\tau_{\text {box }}$ for simulated real samples (Gayeski, et al., 2008).

Figure 7: Comparison of the correct spectral transmittance of a sample $\left(\tau_{\text {sample }}\right.$ and $\left.\rho_{\text {sample }}\left(-^{-}-\right)\right)$with its approximation as a step curve (quasi-spectral estimations $\tau_{\text {box }}$ and $\rho_{\text {box }}(-)$ ) and with the refined estimation results $\left(\tau_{\mathrm{k}}\right.$ and $\rho_{\mathrm{k}}(--)$, k being the point of truncation). (a) combination of Schott filters measured in transmission: GG495 (2 mm) and BG7 (2 mm) (b) V-kool applied film measured in reflection.

Figure 8: (a) Total raw spectral sensitivity of CCD camera, as compared with $V(\lambda)$. (b) Modified spectral sensitivity of CCD camera resulting from a weighted sum of the individual red, green and blue channels (shown in weighted form) and from an additional pair of filters.

Figure 9. From filtering the incoming beam to visualizing transmitted or reflected light distributions in a single image. (a) light source, filter wheel and sample port (b) aluminum coated acrylic hemi-spheroid on Heliodome's rotating support (c) image captured by the camera when measuring the bidirectional transmission distribution function of bubble-wrap within filterband 2. (d) Limit sample size was tested with reflectance measurements of a diffusing sample for $3 \mathrm{~cm}, 5 \mathrm{~cm}$ and $10 \mathrm{~cm}$ sample diameter. (e) HOE transmission image at $40.8 \mathrm{~ms}$ for unfiltered beam, (f) HOE transmission images for filters 1 to 8 .

Figure 10. (a) Flow-chart describing the general image processing method (b) photometric solid for BRDF (white paper, incidence $\left(0^{\circ}, 90^{\circ}\right)$ ) (c) radiometric solid for BTDF (holographic film, incidence $\left(45^{\circ}, 80^{\circ}\right)$. 


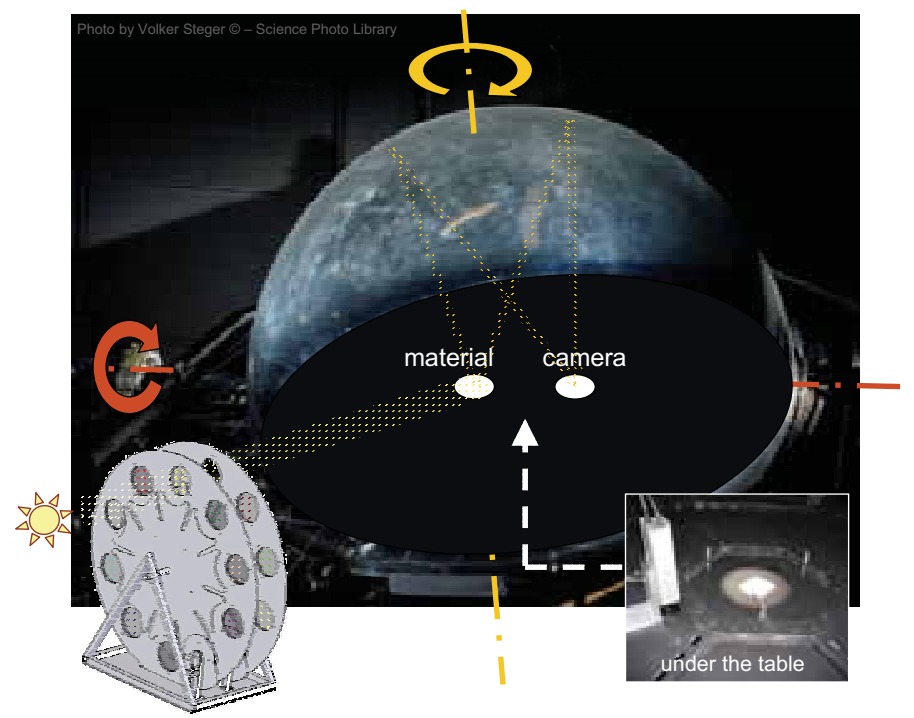

Figure 1: Functioning principle of the Heliodome: The incident light beam reaches the material sample (at one focal point), from which reflected or transmitted light gets reflected once off of the hemi-spheroid to the digital camera (at the other focal point). Note: enhancement illumination was added under the dome for picture quality. 

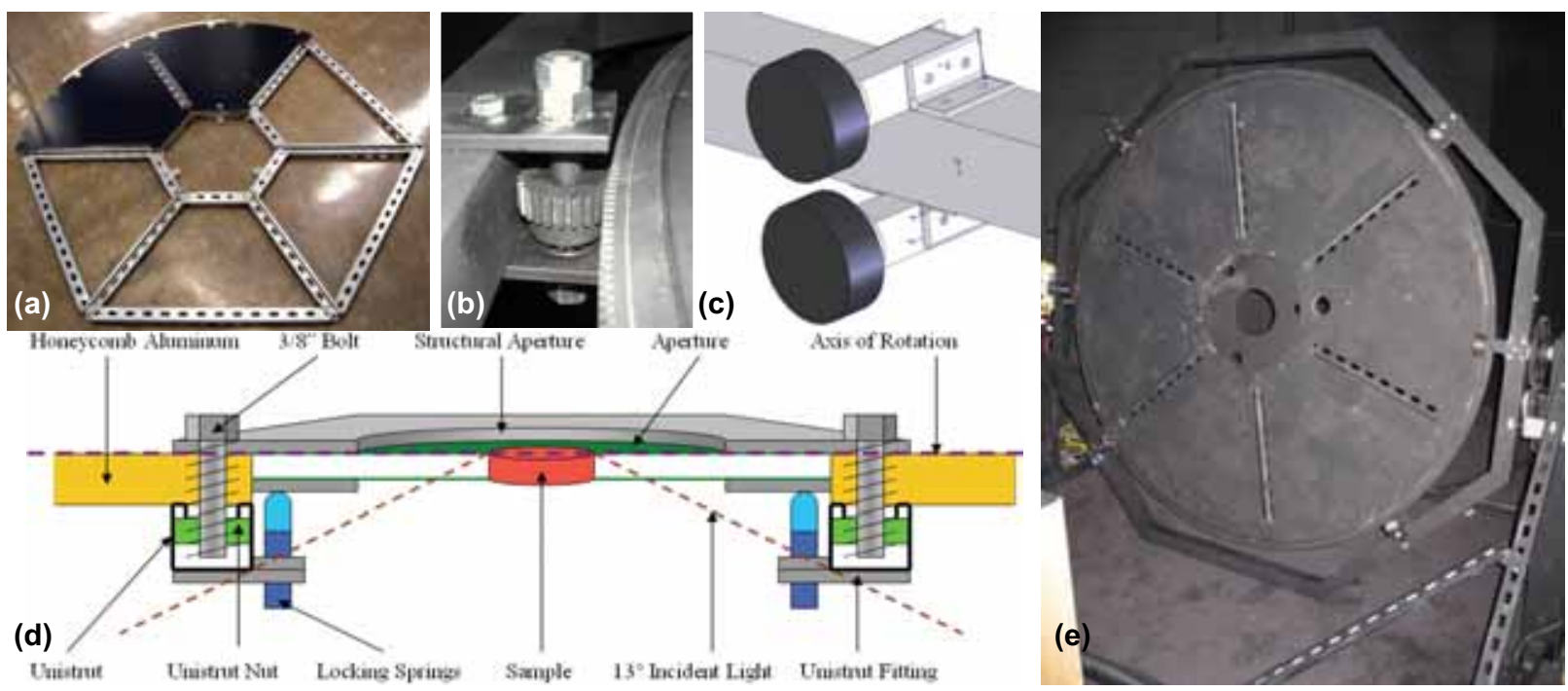

Figure 2. The Heliodome's mechanical support. (a) Assembly of the Table (b) Close-up of Spur Gears (c) Solid Model of Ball Bearing Rollers (d) Construction of Sandwich Locking Mechanism (e) Heliodome supporting table. 

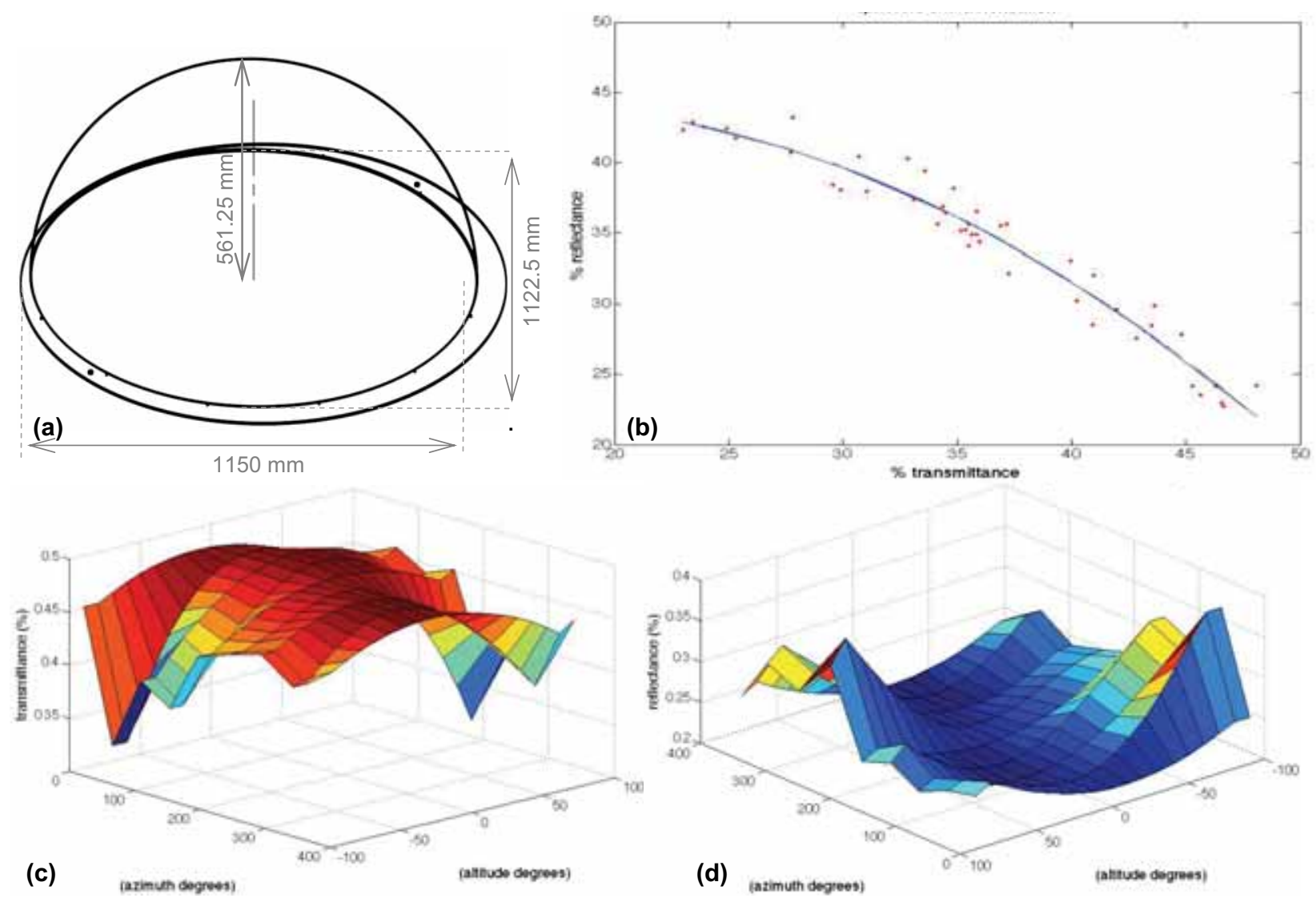

Figure 3. The hemi-spheroid's geometric and optical properties. (a) Major and minor axes (b) Relationship between reflectance and transmission for $500 \mathrm{~nm}$ light (c) Transmittance of spheroid to $500 \mathrm{~nm}$ radiation at all altitude and azimuth angles (d) Reflectance of spheroid to $500 \mathrm{~nm}$ radiation at all altitude and azimuth angles. 

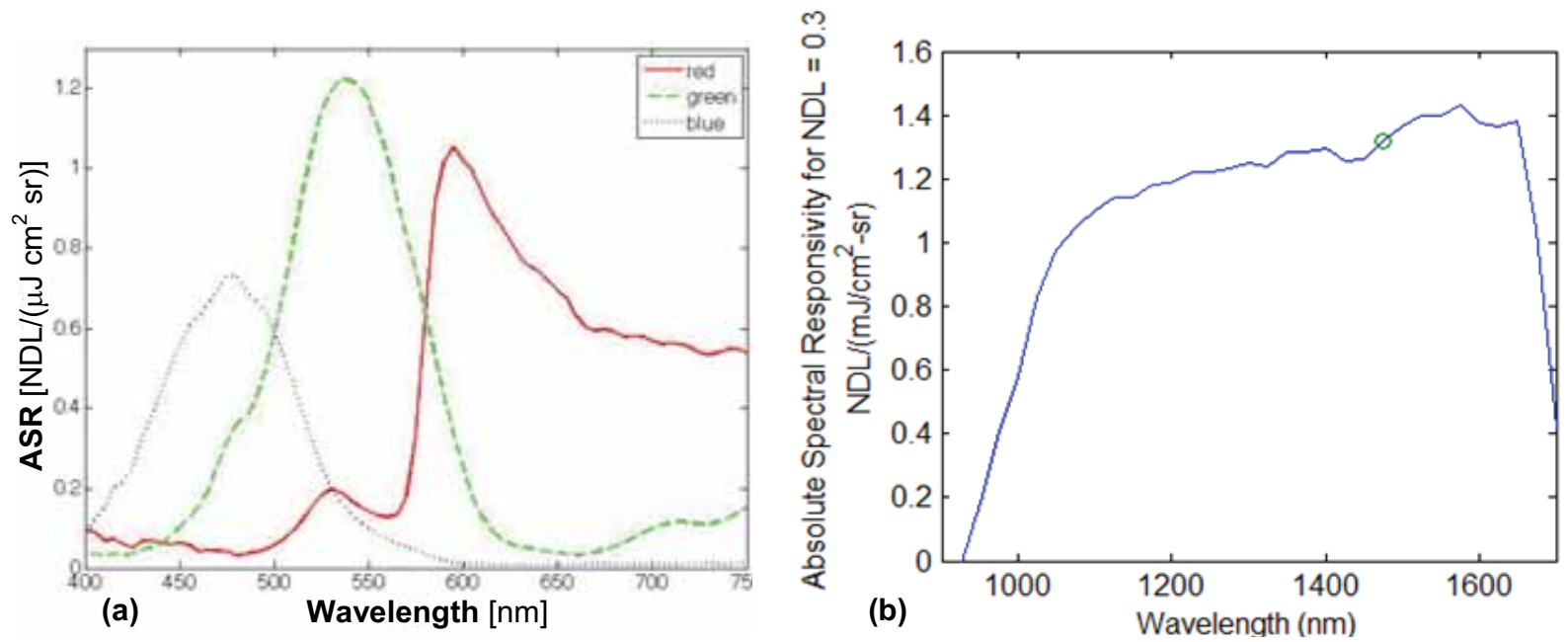

Figure 4: Absolute Spectral Responsivity of the two digital cameras. (a) CCD camera for the visible range, Red Green and Blue channels. (b) InGaAs camera for the near-infrared range, unique channel. 

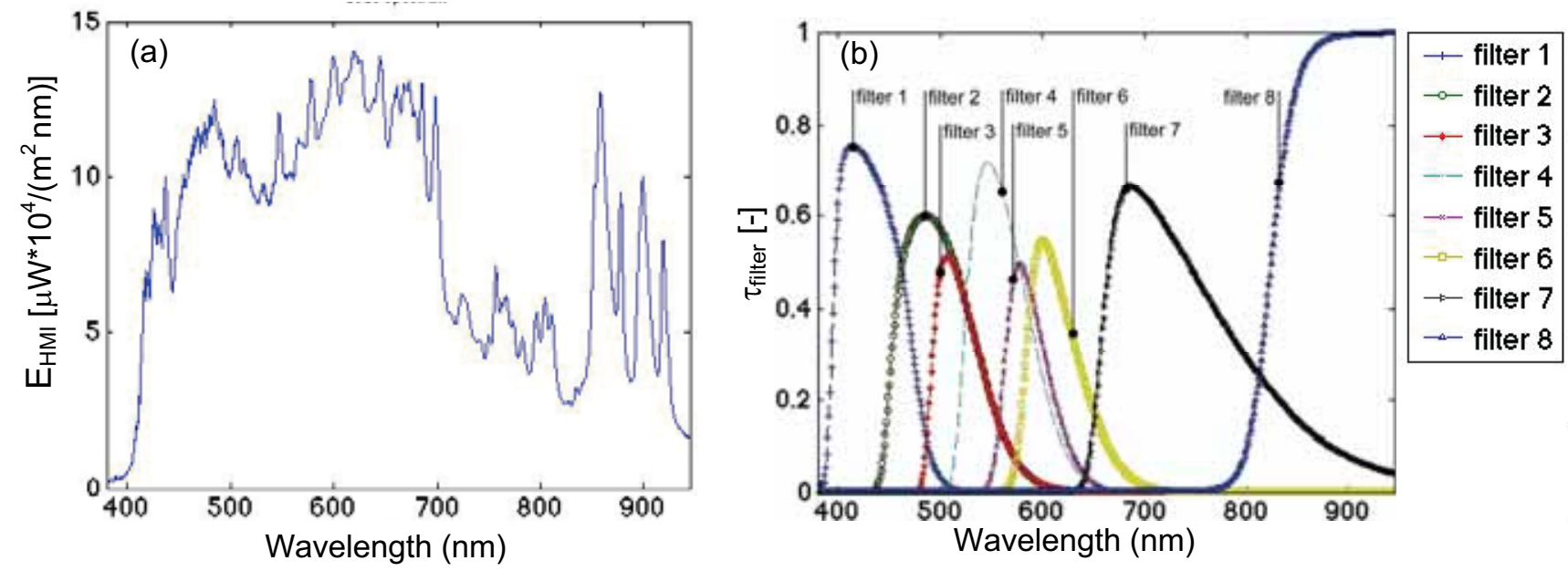

Figure 5. (a) HMI spectrum (b) Filterset spectra over the visible range 

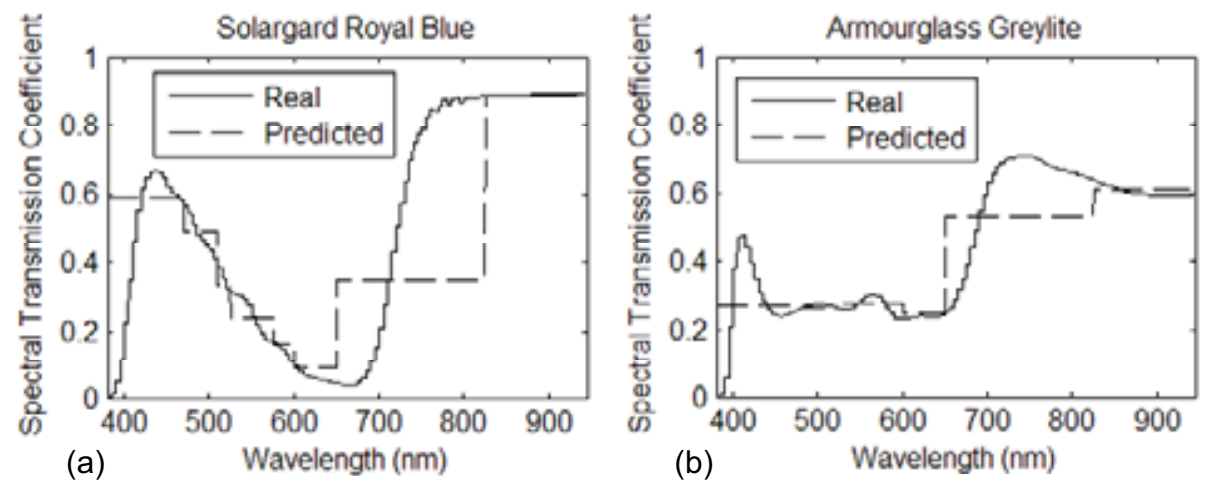

Figure 6. Real and predicted spectral transmittance $\tau_{\text {sample }}$ and quasi-spectral transmittance $\tau_{\text {box }}$ for simulated real samples (Gayeski, et al., 2008). 

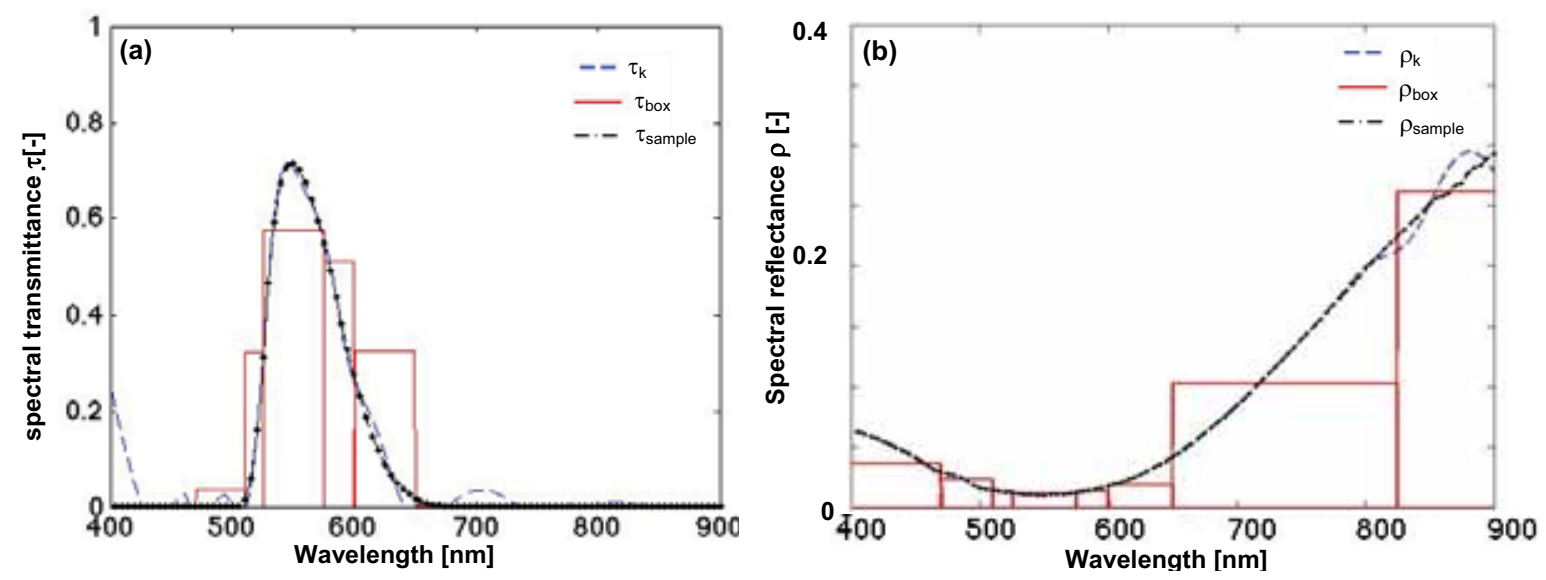

Figure 7: Comparison of the correct spectral transmittance of a sample $\left(\tau\right.$ sample and $\left.\rho_{\text {sample }}\left(-^{-}-\right)\right)$with its approximation as a step curve (quasi-spectral estimations $\tau_{\text {box }}$ and $\rho_{\text {box }}(-)$ ) and with the refined estimation results $\left(\tau_{\mathrm{k}}\right.$ and $\rho_{\mathrm{k}}(--), \mathrm{k}$ being the point of truncation). (a) combination of Schott filters measured in transmission: GG495 (2 mm) and BG7 (2 mm) (b) V-kool applied film measured in reflection. 

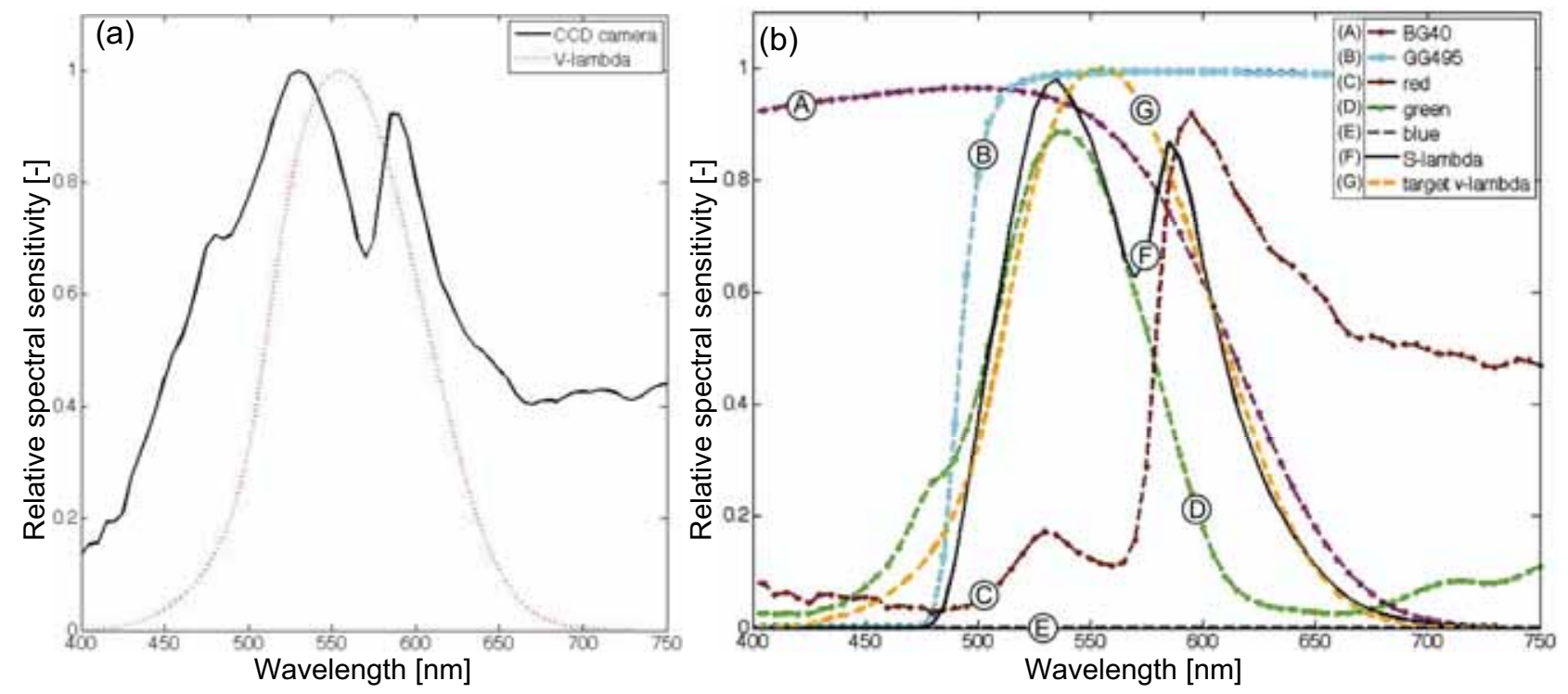

Figure 8: (a) Total raw spectral sensitivity of CCD camera, as compared with $\vee(\lambda)$. (b) Modified spectral sensitivity of CCD camera resulting from a weighted sum of the individual red, green and blue channels (shown in weighted form) and from an additional pair of filters. 


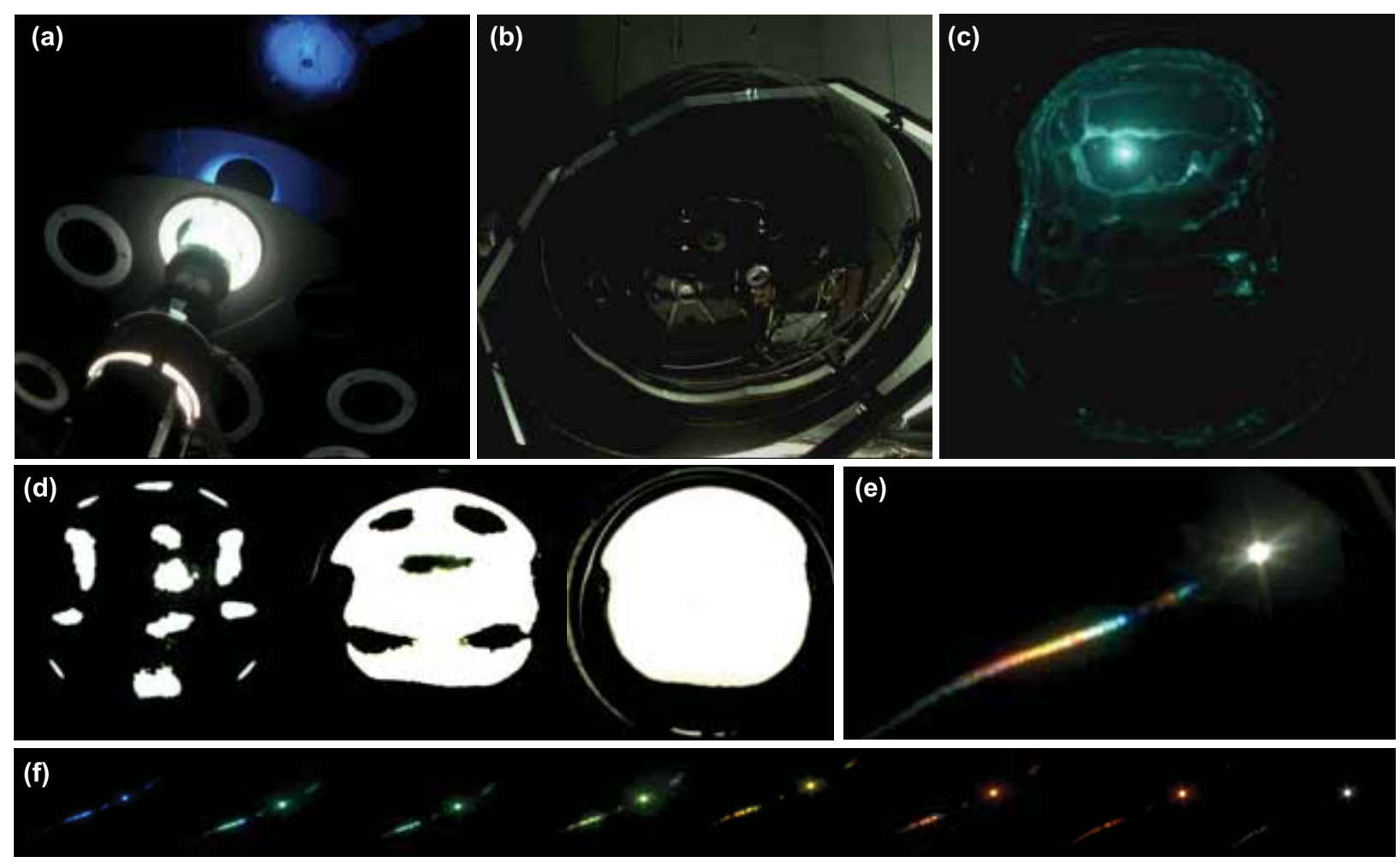

Figure 9. From filtering the incoming beam to visualizing transmitted or reflected light distributions in a single image. (a) light source, filter wheel and sample port (b) aluminum coated acrylic hemi-spheroid on Heliodome's rotating support (c) image captured by the camera when measuring the bidirectional transmission distribution function of bubble-wrap within filterband 2. (d) Limit sample size was tested with reflectance measurements of a diffusing sample for $3 \mathrm{~cm}, 5 \mathrm{~cm}$ and $10 \mathrm{~cm}$ sample diameter. (e) HOE transmission image at 40.8ms for unfiltered beam, (f) HOE transmission images for filters 1 to 8. 


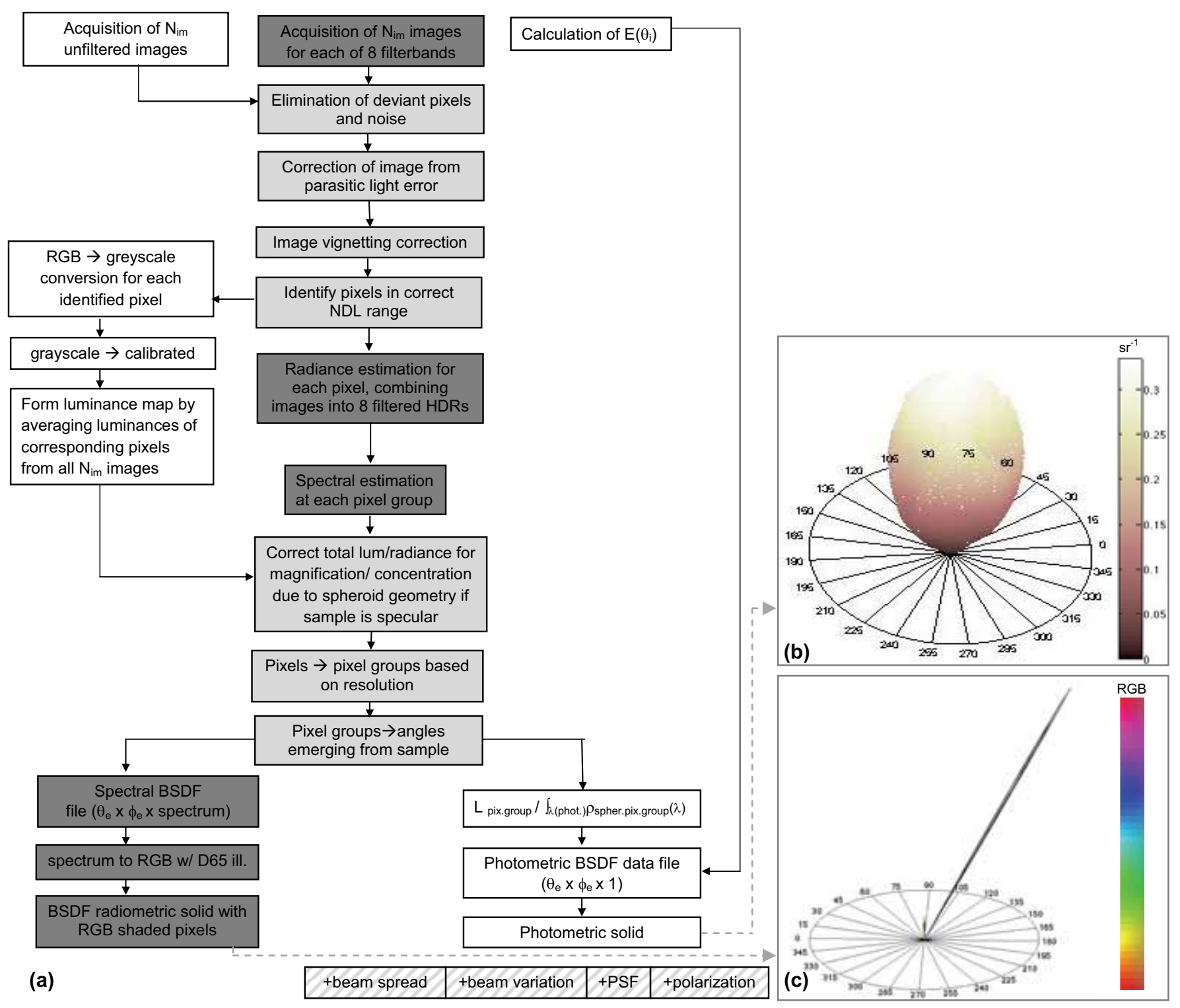

Figure 10. (a) Flow-chart describing the general image processing method (b) photometric solid for BRDF (white paper, incidence $\left(0^{\circ}, 90^{\circ}\right)$ ) (c) radiometric solid for BTDF (holographic film, incidence $\left(45^{\circ}, 80^{\circ}\right)$. 